\title{
Effective modeling of high-energy laboratory-based x-ray phase contrast imaging utilizing absorption masks or gratings
}

Cite as: J. Appl. Phys. 128, 214503 (2020); https://doi.org/10.1063/5.0024475

Submitted: 08 August 2020 . Accepted: 14 November 2020. Published Online: 02 December 2020

(D) Ian Buchanan, (D) Gibril Kallon, Thomas Beckenbach, (i) Joachim Schulz, (D) Marco Endrizzi, and (D) Alessandro Olivo
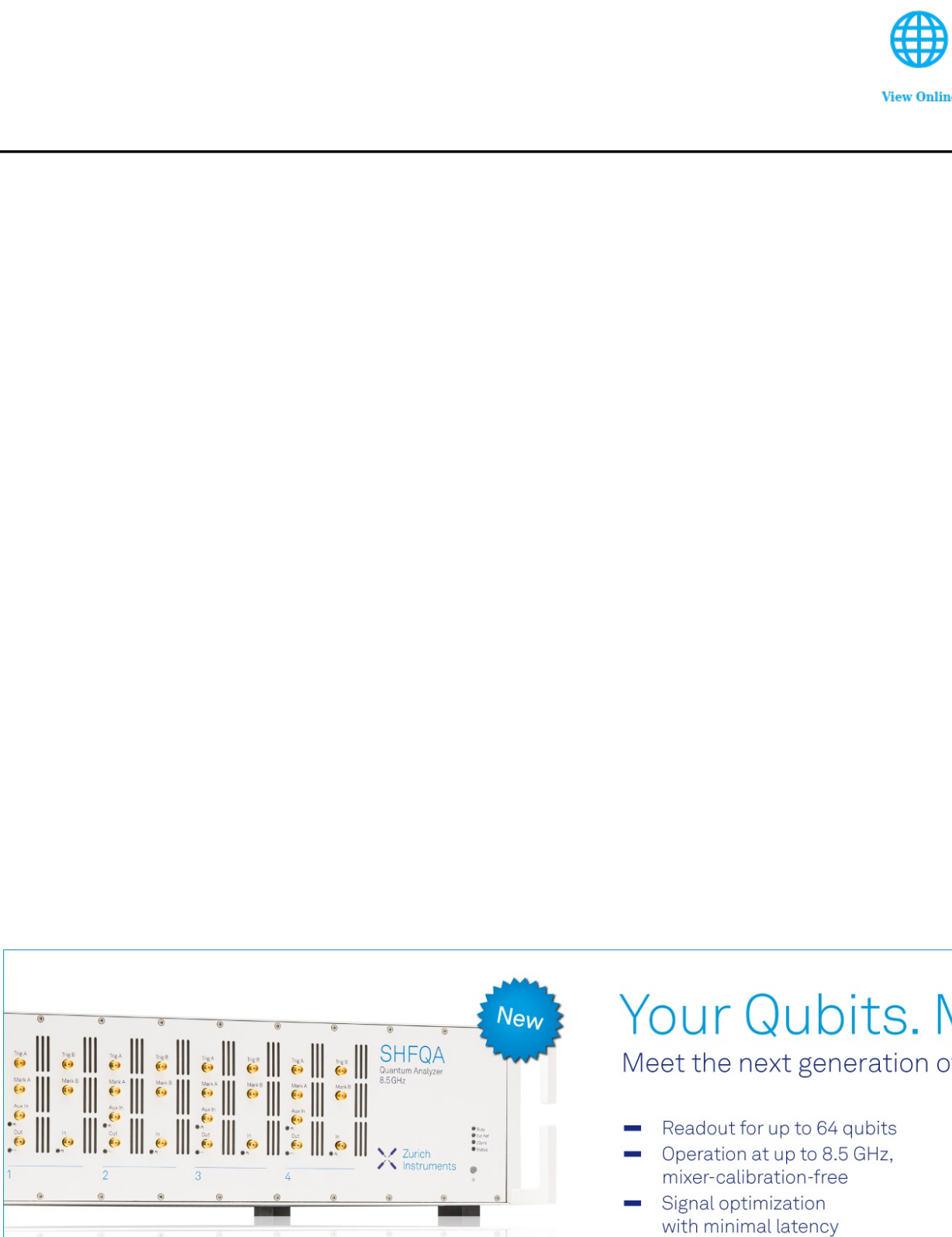

- Readout for up to 64 qubits

- Operation at up to $8.5 \mathrm{GHz}$, mixer-calibration-free

- Signal optimization with minimal latency
Find out more

Zurich Instruments 


\title{
Effective modeling of high-energy laboratory-based $x$-ray phase contrast imaging utilizing absorption masks or gratings
}

Cite as: J. Appl. Phys. 128, 214503 (2020); doi: 10.1063/5.0024475

Submitted: 8 August 2020 . Accepted: 14 November 2020 .

Published Online: 2 December 2020

Ian Buchanan, ,a) (D) Gibril Kallon, ' (D) Thomas Beckenbach, ${ }^{2}$ Joachim Schulz, ${ }^{2}$ (D) Marco Endrizzi, ${ }^{1}$ and Alessandro Olivo ${ }^{\top}$

\author{
AFFILIATIONS \\ ${ }^{7}$ Department of Medical Physics and Biomedical Engineering, University College London, Gower Street, London WC1E 6BT, \\ United Kingdom \\ ${ }^{2}$ MicroWorks GmbH, Schnetzlerstraße 9, 76137 Karlsruhe, Germany
}

${ }^{a)}$ Author to whom correspondence should be addressed: ian.buchanan.15@ucl.ac.uk

\begin{abstract}
Model refinements for the edge illumination x-ray phase contrast imaging method have been developed to improve simulation accuracy for high energy, polychromatic beams. High-energy x rays are desirable in imaging due to their penetrative power and, for biological samples, their lower dose deposition rate. Accurate models of such scenarios are required for designing appropriate imaging systems and to predict signal strength in complex settings such as clinical imaging or industrial quality assurance. When using optical components appropriate for high-energy $\mathrm{x}$ rays in a non-synchrotron setting, system performance was observed to deviate from that predicted by existing models. In this work, experimental data utilizing increasing thicknesses of a known filter material are used to illustrate the limitations of existing models and as validation for the new modeling features. Angular filtration of the cone beam was observed to be the most significant effect; however, specific features of the source and detector are also shown to affect system performance. We conclude by showing that a significantly improved agreement between experimental and simulated data is obtained with the refined model compared to previously existing ones.
\end{abstract}

Published under license by AIP Publishing. https://doi.org/10.1063/5.0024475

\section{INTRODUCTION}

$\mathrm{X}$-ray phase contrast imaging (XPCi) methods allow us to see things that are classically considered "X-ray invisible," with much recent research focusing on the optimization of settings for use in a variety of real-world applications. ${ }^{1-3}$ Imaging in pre-clinical and clinical settings has seen much progress, and quality control in industrial manufacturing is another area of growing interest; such applications could gain from the low dose rates and penetrative power afforded by high-energy $\mathrm{x}$ rays. Sensitivity to changes of the $\mathrm{x}$-ray phase, which decrease with energy at a lower rate than absorption $\left(\mathrm{E}^{2}\right.$, compared to $E^{3.5-3.6}$, depending on the energy range in question ${ }^{4}$ ) also result in a significant boost to image contrast. Furthermore, information regarding microstructures within an object or material-even if these features exist on scales smaller than the pixels being used-also becomes accessible, as these structures can induce ultra-small-angle $\mathrm{x}$-ray scattering (USAXS) or dark-field signals. ${ }^{5}$
In laboratory-based XPCi experiments, cone-beam geometries are often used, and due to the heel effect, photons that reach the detector vary in number and mean energy across the field of view (FoV). For small regions in the detector plane, such variations may be neglected, particularly in cases where the accelerating tube voltage is high, as the heel effect diminishes in significance with increasing photon energy. A source anode's take-off angle determines by how much the beam is inherently filtered and thus what $\mathrm{kVp}$ may be required to overcome the heel effect; shallower angles require higher $\mathrm{kVp}$ settings. In cases where Edge Illumination (EI) masks or Grating Interferometry (GI) gratings are placed in the beam path, an increasing tube voltage requires that the thickness of such optics' absorbing sections also be increased, either to maintain high-attenuation or a specific phase shift, as appropriate. Fabrication of optics with sufficiently high aspect ratios is nontrivial, ${ }^{6,7}$ and, while still only hundreds of microns thick, such 
structures will increasingly attenuate the $\mathrm{x}$-ray beam as it becomes less parallel to the column walls. Additionally, $\mathrm{x}$-ray detector efficiencies typically decrease with increasing photon energy, and detector energy responses are rarely uniform; therefore, prediction of signal to noise ratios (SNRs) becomes a complex exercise. However, modeling of such scenarios is essential to predicting real system performance and, therefore, optimizing system designs. Parameters such as scan times for highly attenuating samples or optimal filtration levels to maximize dose efficiency are two examples of experimental settings that could also be optimized.

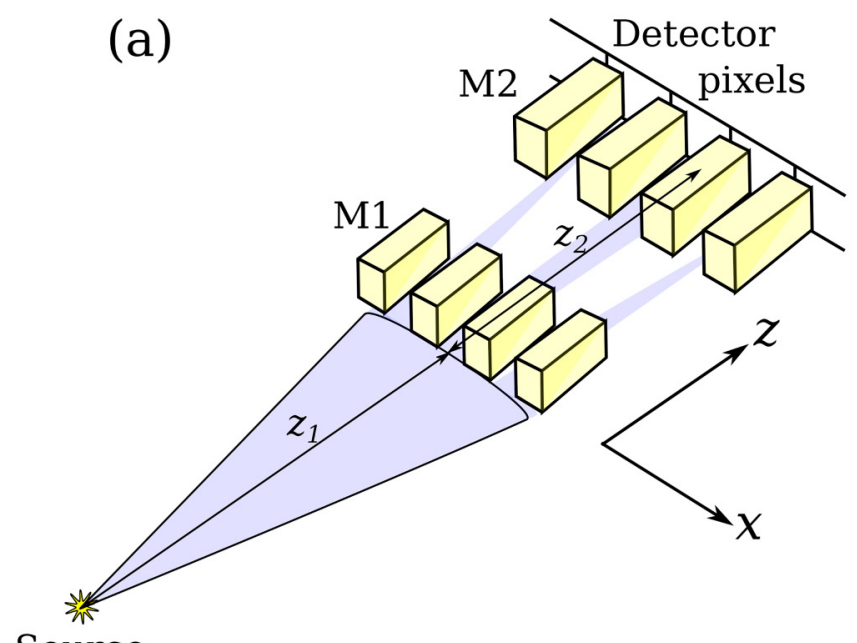

Source

(c)

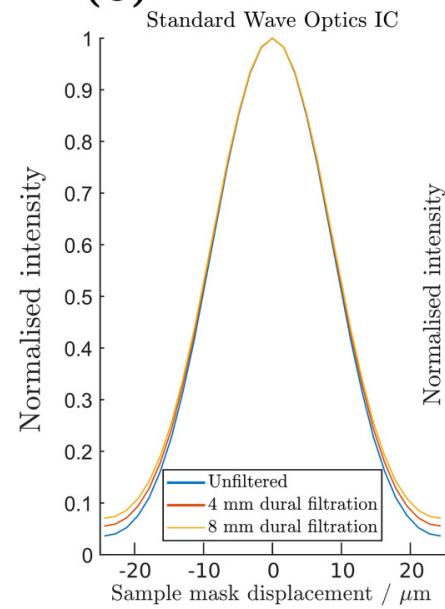

(d)

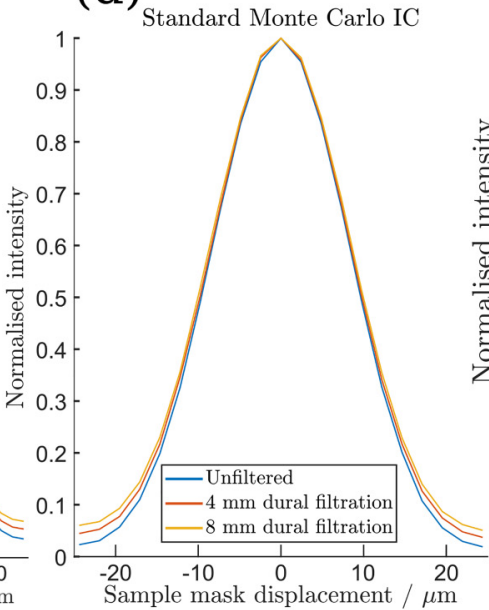

The EI scheme used in this work is shown in Fig. 1(a), with angular filtration by the pre-sample mask, M1, being exaggerated for ease of visibility. The cone beam produced by a low-coherence source is split by M1 into numerous beamlets; these are then incident upon the detector mask, M2, whose pitch is the same as the detector's and M1's after magnification. The $x$ position of M1 can be adjusted to partially illuminate apertures of M2. In such a position, deviations of a beamlet by sample refraction result in more or less of the beamlet being integrated by the detector pixel. Recording the intensity as M1 is translated across a full period allows individual beamlet profiles to be measured. Sample (b)

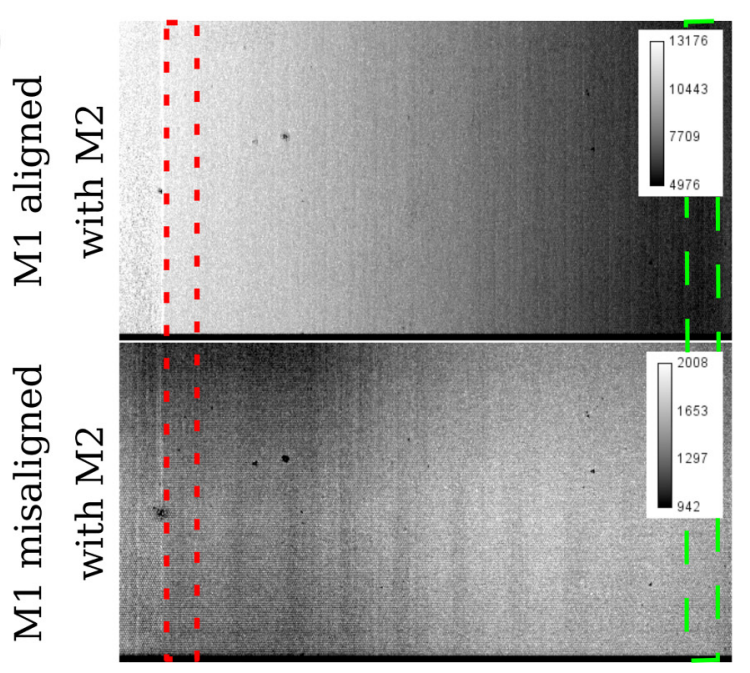

(e)

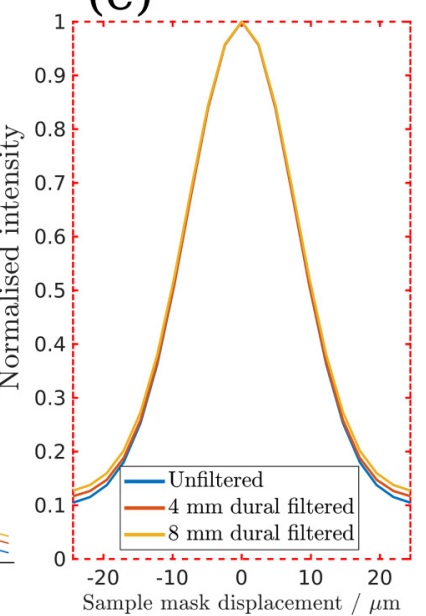

(f)

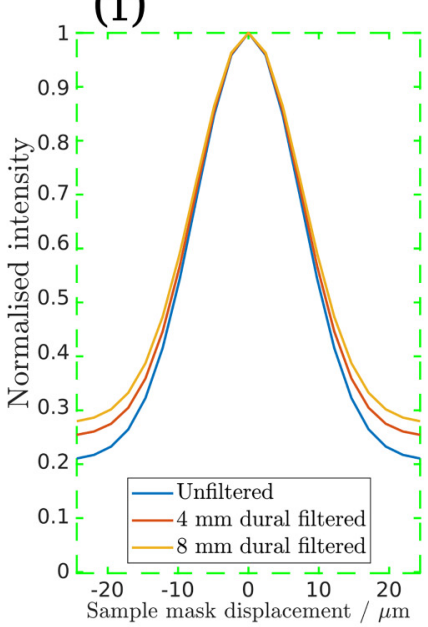

FIG. 1. Edge illumination scheme and data, from experiment and simulations. (a) The El scheme, with exaggerated angular filtration effects. (b) The field of view (520 horizontal pixels) when M1 is aligned (top) and misaligned (bottom) with M2. (c) and (d) show ICs for a $60 \mathrm{kVp} \mathrm{W}$ spectrum without filtration and for those with 4 and $8 \mathrm{~mm}$ dural filters applied, simulated using the previous wave optics and Monte Carlo models, respectively. (e) and (f) show the experimental versions of the same curves extracted near the optical axis [red box in (b)] and at roughly $1^{\circ}$ from the axis [green box in (b)]. A significant difference can be observed both between the sets of experimental curves and with respect to their simulated counterparts. 
attenuation decreases average intensity, refraction shifts the center of gravity, and USAXS broadens the profile. ${ }^{8}$ Fitting algorithms of beamlet profiles, or illumination curves (ICs), usually aim to extract such information via comparison with a well-sampled reference curve, using at least three sampling points when the sample is in place. Other EI adaptions have been developed where the number of sampling points may be less. ${ }^{9,10}$

Figure 1(b) illustrates how the average flux across the FoV decreases when the masks are aligned (corresponding to the maximum of the IC) and that it increases when they are misaligned (minimum of the IC). The intensity measured at the bottom of the IC when the masks are misaligned is referred to as the offset, and parts (c)-(f) of Fig. 1 show how the previous models fail to reproduce such intensities. In this work, we use simulations and experimental data to establish which high-energy $\mathrm{x}$-ray effects are responsible for previously developed models' inaccuracies, from most to least significant. We achieve this by incorporating successive layers of complexity to existing EI-XPCi models based on both wave-optics $(\mathrm{WO})^{11}$ and Monte Carlo $(\mathrm{MC})^{12}$ implementations, which have already been validated for lower x-ray energies. The simulation results are verified, where possible, using experimental measurements of wire phantoms, which were acquired using an increasingly filtered $60 \mathrm{kVp}$ tungsten beam. The filter material was dural, an alloy consisting primarily of aluminum (usually 95\%), copper (up to 5\%), and trace amounts of other metals such as manganese and magnesium. Sheets of $0.5 \mathrm{~mm}$ thick dural were layered in front of the $\mathrm{x}$-ray tube window to achieve mean energies in the range of $26-48 \mathrm{keV}$. Spectral and refractive index data were generated using TASMICS $^{13}$ and xraylib, ${ }^{14}$ respectively. As two models are being developed in parallel, the structure of this paper is to present an effect and then to explain how it is implemented in each model in turn. The refined models are expected to be of interest to users of both EI and GI, as they will likely be useful for designing better experimental optics for high $\mathrm{x}$-ray energies.

\section{THE SYSTEM}

The experimental setup consisted of a Rigaku MultiMax-9 source with a rotating tungsten anode whose take-off angle was $6^{\circ}$. When required, filters were placed immediately after the source window. The masks, placed $1.6 \mathrm{~m}$ and $2 \mathrm{~m}$ downstream of the source, were manufactured by microWorks $\mathrm{GmbH}^{7}$, with pitches $p_{1}=49 \mu \mathrm{m}$ and $p_{2}=61 \mu \mathrm{m}$ and apertures $w_{1}=12 \mu \mathrm{m}$ and $w_{2}=18 \mu \mathrm{m}$. The gold septa thicknesses in M1 and M2 were $310 \pm 2 \mu \mathrm{m}$ and $319 \pm 2 \mu \mathrm{m}$, respectively, with densities of $90 \pm 1 \%$ that of solid gold due to slightly non-uniform electroplating; ${ }^{15-17}$ and the substrate of each mask was a $525 \mu \mathrm{m}$ thick layer of silicon. An energy resolving and photon counting detector (Pixirad PANalytical, Malvern UK) ${ }^{18}$ was placed immediately downstream of M2. It consists of a $650 \mu \mathrm{m}$ thick CdTe sensor layer and $62 \mu \mathrm{m}$ square pixels; it was operated at a temperature of $-20^{\circ} \mathrm{C}$, achieved by Peltier cooling. The detector consists of two readout chip arrays; however, only one of these was used due to there being a slight inclination between the arrays; thus, for a given set of EI masks, alignment with pixel columns could only be achieved for one module at a time. To prevent ice crystals from forming, dry, inert air, supplied by a pressurized canister, was constantly passed over the active area. Wire samples were mounted as closely as possible to M1 and translated in sub-pixel steps to achieve highly sampled image profiles.

\section{ANGULAR FILTRATION}

EI at high $\mathrm{x}$-ray energy requires masks with relatively thick absorbing septa (usually gold or tungsten). The mask periods are on the order of tens of microns, while the gold column thicknesses will need to be several hundreds of microns thick, meaning that the aspect ratio of these structures is relatively high; $\sim 8$ for both masks. The columns are adhered to a non-scattering substrate and are either stand-alone or interspersed with a weakly attenuating resist material such as epoxy resin or SU-8, which acts as support for the high aspect ratio structures. ${ }^{6}$ For the experiments reported and simulated in this work, angular filtration due to the septa of both masks reduced the flux by more than $50 \%$ across the FoV when they were aligned, as shown in Fig. 1(b). This is due to photons approaching the masks at an angle to the column walls and being intersected by the septa corners, both at the base and top. A secondary effect of this is that the average energy of the beamlets is expected to increase with the angle of photon approach, though this is not examined in detail here.

\section{A. Monte Carlo}

McXtrace ${ }^{19}$ was adapted for EI simulations by Millard et al.: ${ }^{12}$ all previous measures aimed at attaining computational efficiency, such as formatted look-up tables and changes in photon trajectories by Snell's law, are maintained in these updates. Modeling of angular filtration on a large scale is simpler in an MC-type environment, where mask components can be upgraded from a simple transmission function to a pseudo-three-dimensional object. In the first instance, a photon arriving at the mask component is attenuated or not depending on whether it arrives at an aperture or septa, determined by Eq. (1):

$$
T(x)=\left\{\begin{array}{c}
1 \text { if } \cos \left(\frac{2 \pi x}{p}\right) \geq \cos \left(\frac{\pi \omega}{p}\right) \\
e^{-\mu t}, \quad \text { otherwise }
\end{array}\right.
$$

where $x$ is the lateral position in the mask plane, $p$ the mask pitch, $\omega$ the aperture width, $\mu$ the linear attenuation coefficient of the septa, and $t$ the septa thickness. In McXtrace, photon weights are used instead of a binary absorption/transmission model so that all computations contribute to the final image statistics. All photons are initially assigned the same weight, which is subsequently reduced according to the Beer-Lambert law for each photon's optical path through different materials.

In the updated mask component, the period still determines whether a photon arrives at an aperture, but propagation through the component and the subsequent path lengths through any absorbing features are used to adjust photon weights before leaving the component. A 3D model of the entire mask is unnecessaryphotons entering the component will not traverse an entire mask period before exiting unless the angle of incidence is very large 
$\left(>81^{\circ}\right.$ for the masks considered here). Rather, once inside the component, it is cheaper both computationally and in terms of memory usage to determine the relative location and orientation of the nearest septa walls and hence whether the photon will intersect any of them before exiting.

Upon reaching any boundary in the mask component, Snell's law is used to update the photon's trajectory, and this is repeated until the photon has cleared the component entirely. The change in the path length for a photon moving from aperture to septa is negligible for small angles but becomes larger as the angle of incidence grows. For the range of mean energies being considered here, the critical angle for photons impinging upon gold is between $0.2^{\circ}$ and $0.1^{\circ}$, below which the photon would normally be reflected. However, given that the manufacturer's estimates of surface roughness are on the order of $15 \mathrm{~nm}$, the likelihood of reflection becomes exceedingly small $(\mathrm{P}<0.1 \%$, for photons of $\mathrm{E}>20 \mathrm{keV}$ and whose angle of incidence is $\left.>0.05^{\circ}\right) ;^{20}$ therefore, in our model, photons arriving at or below the critical angle are simply absorbed for computational efficiency. In the case where simulations are run assuming perfectly smooth, reflecting septa walls, the change in outputs was found to be negligible. The final calculated path lengths through septa and resist, together with the energy-specific attenuation coefficients, are used to adjust each photon's weight. The process is illustrated schematically in Fig. 2(a).

\section{B. Wave optics}

The wave optics model developed by Vittoria et al. ${ }^{11}$ uses fast Fourier transforms to implement the Helmholtz propagator. Both masks, and any samples modeled, act on a wavefront as complex transmission functions, while blurring due to the detector point spread function (PSF) and an extended source are implemented by convolving the intensity profile with appropriate Gaussians. Polychromatic simulations are constructed via spectrally weighted sums of monochromatic data. Simulating large fields of view without aliasing can be challenging, though high frequency filters can be used to mitigate this. ${ }^{21}$ A more prosaic task is to develop a mask transmission function, which intrinsically filters the beam as $|x|$ increases; this could be achieved by calculating the optical path length through mask's contents for every sampled angle. An efficient approach, used here, is to use distinct transmission functions, valid within small angular ranges, to simulate parts of the FoV; pixel data from multiple simulations are then combined to create the full FoV.

Experimental data show that loss of flux due to angular filtration is slightly greater than $50 \%$ over 500 pixels; thus, wavefronts spanning approximately ten pixels are simulated at a time. For given subsets of the FoV, an appropriate mask transmission function is determined by the process shown in Fig. 2(b): a matrix of $1 \mathrm{~s}$ and $i$ 's, indicating the presence of gold or photoresist voxels, respectively, is constructed and rotated to the required angle. The projected thicknesses over a single period are found by integration. The WO model uses a single coordinate geometry corresponding to the M1 plane, and operations occurring at the detector plane are demagnified to enable this; thus, it is necessary to construct individual mask matrices for both M1 and M2, as the ratio of aperture width to period is different in each case.

\section{PIXIRAD DETECTOR RESPONSE}

Pixirad is equipped with a lower and upper energy threshold. For experiments, the lower was set to $10 \mathrm{keV}$, which
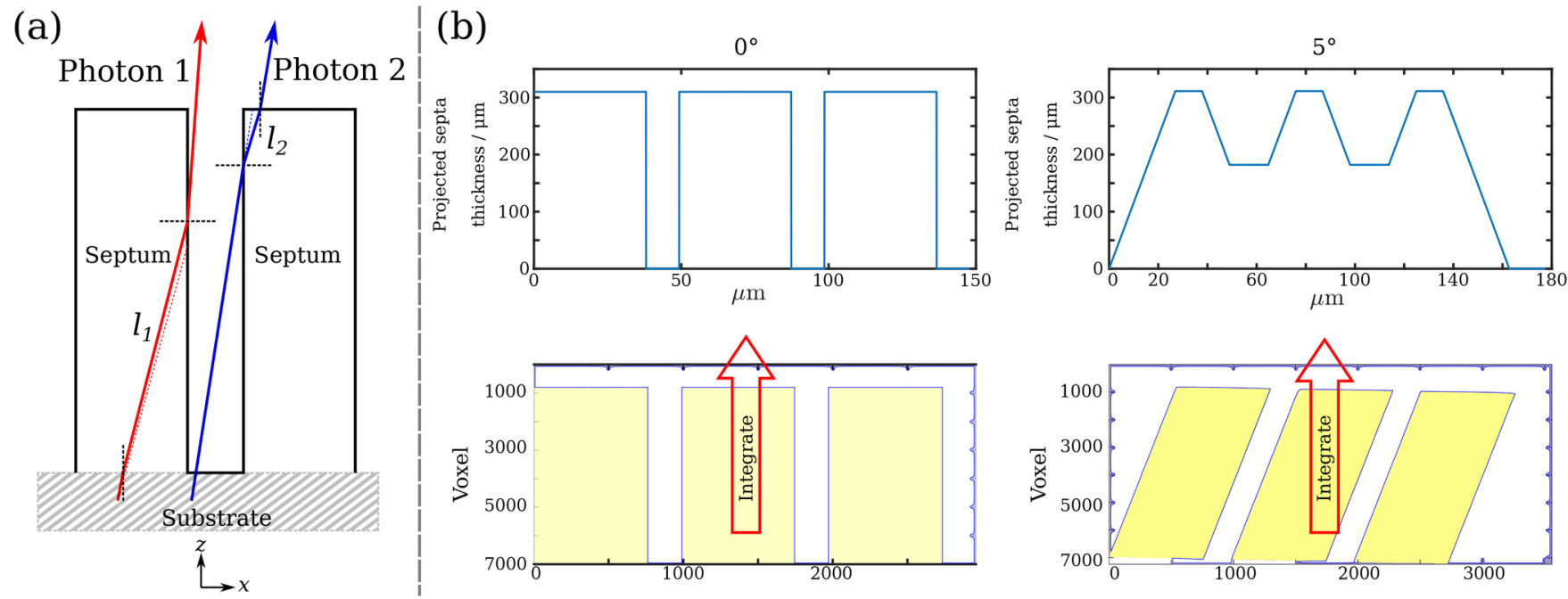

FIG. 2. Schematic of angular filtration being implemented in the models. (a) MC: the path length through the substrate is calculated geometrically, and Snell's law is used to determine the distance traveled through absorbing septa. (b) WO: the transmission function is determined by vertical integration of a mask image that is rotated to the angle of incidence made by photons approaching a certain region of the detector ( $5^{\circ}$ is an extreme example used for demonstration of the changing profile). 
eliminated noise when the beam was off; the upper was set to $70 \mathrm{keV}$ to prevent electronic pileup. Multi-counting, where a single photon is responsible for multiple counter increments within the detector, is most likely to occur near pixel edges; ${ }^{22}$ it has a smoothing effect on image noise but is usually negligible compared to the number of primary counts. In addition, for multi-counting to occur, the photon in question must also have energy at least greater than twice the lower energy threshold.

In low x-ray energy applications, EI's design ensures that multi-counting occurs to a very little extent, as the septa of M2 straddle pixel boundaries, thus blocking photons from the regions most susceptible to multi-counting. However, in the high-energy case, where mask septa may become partially transparent, an unwanted consequence of such mask placement is that photons arriving near pixel edges are predominantly of high energy due to significant gold filtration. Thus, counts measured experimentally are likely to be greater than basic simulations suggest. Figure 1 supports this: even with wavefronts being almost perpendicular to apertures [red box in Fig. 1(b) and ICs in Fig. 1(e)], the measured IC offset is higher than predicted by the standard simulations.

\section{A. McXtrace}

Adjustment of the pixel response in McXtrace is conceptually simple but relatively demanding in terms of memory usage. Normally, each pixel is a defined region in the detector component and photon weights are added as they arrive-in this case, each photon must be tracked more carefully and checked against different energy conditions. A photon interacting with the detector sensor layer produces a delta function cloud of electron-hole pairs that drift toward the anode and cathode, respectively; in order to estimate the radius $r$ of a diffuse electron cloud within the sensor layer, Eq. (2) is used, ${ }^{23}$

$$
r=1.15 d \sqrt{ }\left(2 k_{B} T / q V\right)
$$

where $d$ is the photoconductor thickness $(\sim 650 \mu \mathrm{m}), k_{B}$ the Boltzmann constant, $T$ the temperature in Kelvin, $q$ the electron charge, and $V$ the applied bias voltage $(400 \mathrm{~V})$. Using these values leads to an approximate charge cloud radius of $7.8 \mu \mathrm{m}$. Equation (2) assumes an initial electron cloud as being a delta function; however, the initial diameter is likely to be of a similar magnitude to that calculated here. This radius is thus taken as a lower bound for the electron cloud, and we note that using exact, larger values will have only a third-order effect on the data shown here. We maintain this approximation for the electron cloud radius for all photon energies considered due to them being less than $100 \mathrm{keV}^{24}$

We decided to sub-sample each modeled pixel by a factor of five and established four energy bins for the ranges of $10<\mathrm{E}<22.5$, $22.5<\mathrm{E}<35,35<\mathrm{E}<47.5$, and $47.5<\mathrm{E}<60$. After a simulation has run, pixel groupings are defined for the sub-sampled data, weighted according to how much multi-counting might be induced based on the specific energy bin and then summed to produce the final pixel value, as illustrated in Fig. 3(a).

Low energy photons arriving in pixel corners result in missed events, while high-energy photons are counted up to three times in the final energy bin considered. In the future, this sub-pixel sensitivity and weighting could be performed with arbitrary precision within the detector component since the effectiveness has been verified. (a)

\begin{tabular}{|c|c|c|c|c|}
\hline \multicolumn{1}{c}{$10<\mathrm{E}<22.5$} \\
\hline 0.56 & 0.8 & 0.8 & 0.8 & 0.56 \\
\hline 0.8 & 1 & 1 & 1 & 0.8 \\
\hline 0.8 & 1 & 1 & 1 & 0.8 \\
\hline 0.8 & 1 & 1 & 1 & 0.8 \\
\hline 0.56 & 0.8 & 0.8 & 0.8 & 0.56 \\
\hline
\end{tabular}

\begin{tabular}{|c|c|c|c|c|}
\multicolumn{5}{c|}{$22.5<\mathrm{E}<35$} \\
\hline 1 & 1 & 1 & 1 & 1 \\
\hline 1 & 1 & 1 & 1 & 1 \\
\hline 1 & 1 & 1 & 1 & 1 \\
\hline 1 & 1 & 1 & 1 & 1 \\
\hline 1 & 1 & 1 & 1 & 1 \\
\hline
\end{tabular}

\begin{tabular}{l}
\multicolumn{1}{c|}{$35<\mathrm{E}<47.5$} \\
\begin{tabular}{|c|c|c|c|c|}
\hline 1.4 & 1.2 & 1.2 & 1.2 & 1.4 \\
\hline 1.2 & 1 & 1 & 1 & 1.2 \\
\hline 1.2 & 1 & 1 & 1 & 1.2 \\
\hline 1.2 & 1 & 1 & 1 & 1.2 \\
\hline 1.4 & 1.2 & 1.2 & 1.2 & 1.4 \\
\hline
\end{tabular}
\end{tabular}

(b)
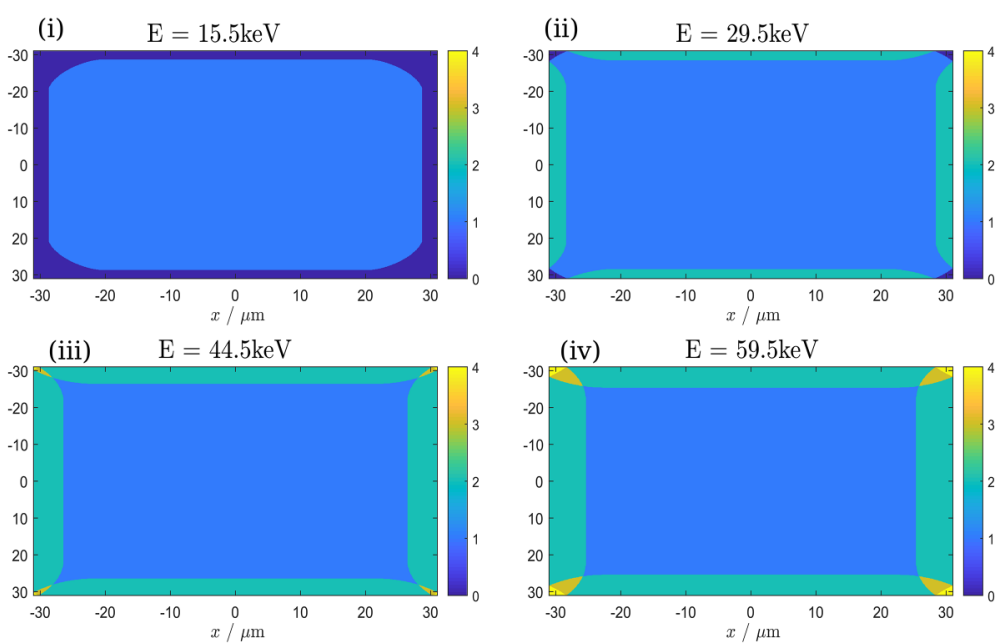

FIG. 3. Multi-counting implementations: Monte Carlo (a) sub-samples the pixel and applies weightings depending on photon energy and WO (b) uses the averaged profile of these pixel images to boost the integrated signal by the correct amount. 


\section{B. Wave optics}

After the wavefront has been propagated from M1 to the detector plane, multiplied by the transmission function of $\mathrm{M} 2$, and blurring convolutions due to source and detector PSF have been performed, a top-hat function-representing the pixel sampling-is convolved with the intensity profile. The average pixel value is then the centrally sampled point, in real space, of the resulting output. Here, the "hat" of the pixel function is replaced with a function that represents the mean over-counting rate as a function of position. This line profile is the average of a $2 \mathrm{D}$ heat-map of multicounting events, examples of which are shown in Fig. 3(b). It is created by considering the integral of a charge cloud, centered in a given voxel, over the originating and adjacent pixels. For each instance, where a pixel's integral exceeds the charge cloud threshold, the weight of that cloud's central sampling position is incremented by one. The charge cloud threshold is determined by the lower energy threshold used in experiments and decreases with increasing photon energy. These integrals only need to be performed once and the heat-maps, or line profiles, can then be saved as a variable to be called upon by later simulations.

\section{VARIABLE SOURCE SIZE AND THE HEEL EFFECT}

Diemoz et al. showed that, within certain focal spot ranges, the sensitivity of EI is strongly dependent on the source width (FWHM, for a Gaussian source), ${ }^{25}$ which affects the width of measured ICs. The variation in the projected focal spot size across the

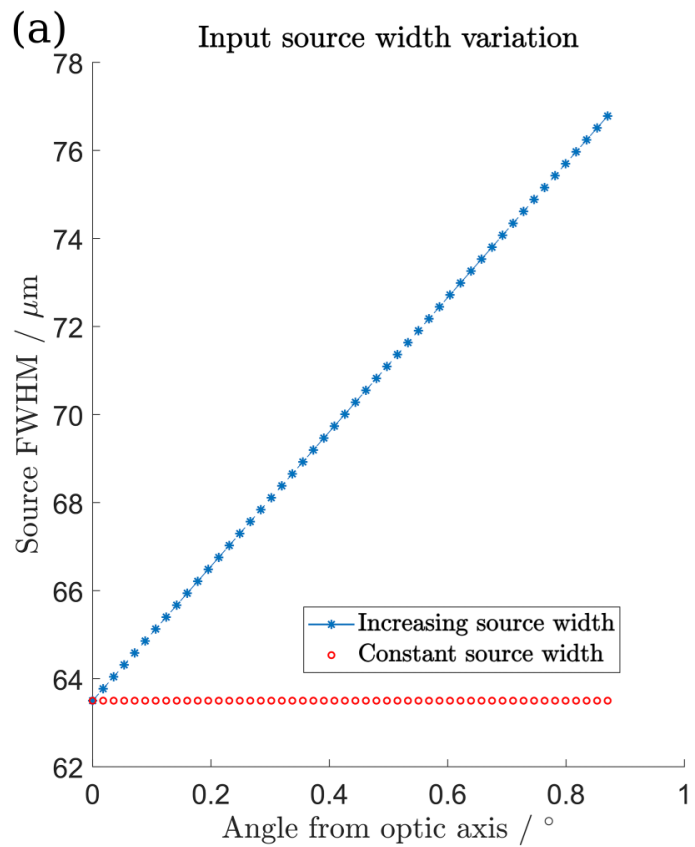

FoV, caused by the use of a slanted anode, therefore, needs to be incorporated in the simulations.

Thus, two effects to consider when analyzing the IC width are

1. The use of an angled anode means that the apparent source size varies depending on the sine of the angle at which it is viewed.

2. As a photon's angle of incidence with septa walls increases, angular filtration narrows the resultant beamlet. This requires accurate knowledge of septa thickness in order to simulate accurately.

In our system, these effects initially appear to cancel one another. However, for angles greater than $0.4^{\circ}$, the first effect dominates, as shown by the increasing IC width across the FoV in Figs. 4(b) and 4(c). As noted in Sec. II, only one module of the detector was used for the EI experiments and the other was used to estimate the location of the optic axis. However, due to the uncertainty of the inclination of this module, there is some uncertainty about where the optic axis would appear for the aligned module. We note that a shift of the experimental curves in Figs. 4(b) and 4(c), by $\sim 0.1^{\circ}$, would improve the match between the simulated and experimental data, though exact matches are not sought here; rather, we seek to illustrate that the observed trends are being correctly modeled.

The heel effect, which changes the average beam energy (as well as the x-ray flux) as a function of emission angle, is due to the varying amount of self-filtration of the source by the tungsten anode. Over the FoV and for the take-off angle considered in this case, the expected variation in the path length through the tungsten
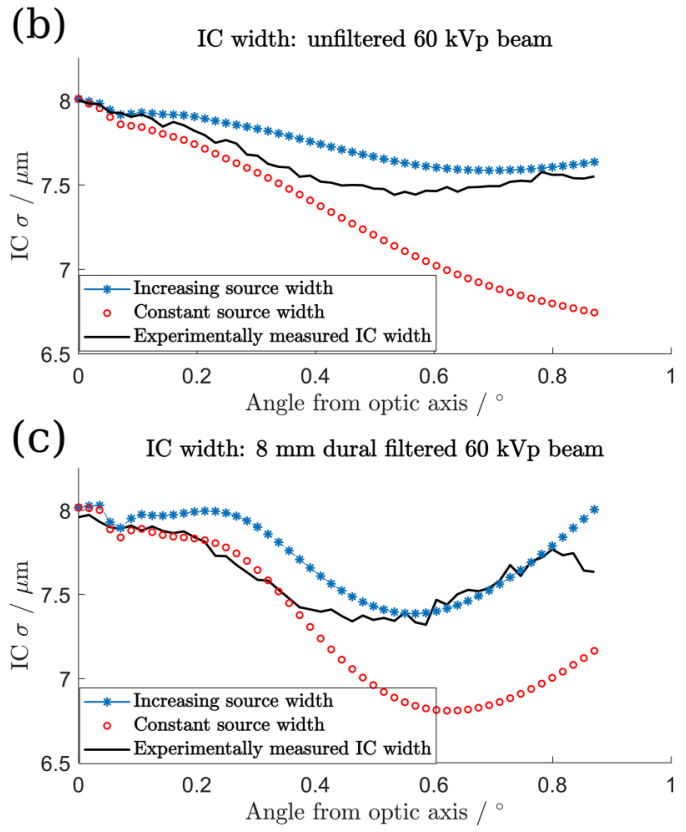

FIG. 4. Comparison of a single source size used in simulations across the field of view with that of an increasing source size. (a) Shows the simulation input values for the source FWHM in each case, while (b) and (c) show the resulting IC width parameters for the unfiltered and $8 \mathrm{~mm}$ dural filtered $60 \mathrm{kVp}$ beam, respectively, along with the experimentally measured widths. 
anode is $\sim 1 \mu \mathrm{m}$. This leads to a negligible variation of mean energy, especially since the beam is subsequently hardened by millimeters of dural in the experiments reported here. However, the potential influence on the photon flux being emitted across the FoV is a potential source of inaccuracy. This latter effect is indistinguishable from angular filtration when the masks are in place but could possibly be corrected for by acquiring a "source-only" image.

\section{A. Monte Carlo}

In McXtrace, the source component takes a FWHM value as an argument: this determines the range from which the points of emission in the source plane are selected. A direction of emission, confined to the detector's solid angle, is then chosen at random. We implemented the appropriate variation in the source size by reversing the order of these operations to first determine the angle of emission. This was used to scale the source distribution according to Eq. (3),

$$
\sigma_{x}=\sigma_{x_{0}} \sin \left(\theta_{0}+\theta\right) / \sin \left(\theta_{0}\right),
$$

where $\sigma_{x_{0}}$ is the initial FWHM parameter as measured along the optic axis and $\theta_{0}$ is the initial take-off angle of the source $\left(6^{\circ}\right.$ in this case). This equation is derived through geometric considerations of the solid angle made by the impact area of the electrons on the anode as seen by the detector as a function of viewing angle; the denominator is in place to ensure that, along the optic axis, $\sigma_{x}$ has a fixed starting value.

The heel effect is applied using the same method: once the angle of emission has been randomly generated, it is used to calculate the projected anode thickness that attenuates the photon. This thickness varies between $5 \mu \mathrm{m}$ and $4 \mu \mathrm{m}$, decreasing as the angle of emission increases. Such an approach is useful for modeling the reduction of flux and the change in average energy simultaneously. Importantly, as the beam softens with increasing emission angles, attenuation due to angular filtration in the masks increases.

\section{B. Wave optics}

Source blurring is simulated by a convolution in the detector plane between a Gaussian and the intensity profile produced by a point source. A simulation with a Gaussian source of variable width is achieved by piecewise simulations of small pixel regions, each using Eq. (3) to determine the FWHM for given angles of emission. As shown by Figs. 4(a)-4(c), the variation in the source size only becomes significant for angles greater than $0.4^{\circ}$; up to this point, the decrease in the effective aperture width dominates the IC width parameter. The heel effect is modeled as described above.

\section{POSSIBLE EFFECTS OF VARIATIONS IN SEPTA DENSITY AND AIR SCATTERING}

Density measurements of the absorbing septa were performed by weighing and optically measuring the dimensions of a small mask region excised by diamond cutting tools. It was not possible to experimentally measure how the density of each septum varied throughout the lamellae. WO simulations, with which it is trivial to modify the density profile, demonstrate that the IC offset is affected by doing so, particularly for photons traveling along the optical axis. As the possible density profiles, both horizontal and vertical, are infinite in number, an exact setting that produced IC offsets as seen in the experiment was not sought. Instead, three simple cases were considered:

- a uniform, $90 \%$ density throughout the septa,

- a sinusoidal variation in the horizontal density throughout, whose mean is $90 \%$ and has a $100 \%$ density along septum walls,

- a sinusoidal variation as above, but with a $80 \%$ density along septum walls,

as shown in Fig. 5(a). Part (b) of this figure shows the IC offset intensity, relative to the peak height, for an unfiltered $60 \mathrm{kVp}$ beam when only these effects are added to the model. This variation in offset is of interest, as it affects the maximum gradient of IC slopes and thus the system's sensitivity to phase effects. However, the effect appears limited to regions close to the optic axis, and the increase in the offset due to the posited density variations is, at most, $1 \%$. As no oscillations in the experimentally measured offset can be seen, perhaps obscured by noise, the effect of variable septa density can be considered an uncertainty in simulated offset values when considering pixels close to the optic axis. Up to what number of pixels away from the axis this is true will depend on septa thicknesses.

Scattering of $\mathrm{x}$ rays in air is also a mechanism by which ICs may be broadened. To some extent, the masks used in EI can be expected to act as anti-scatter grids, though to what extent is unknown. In order to determine this, the scattering simulation techniques developed by Busi et al. ${ }^{26}$ were implemented in the MC model. An "air-block" component is placed between the source and M1 and between M1 and M2. Photons in these components are propagated by discrete amounts, and after each discrete
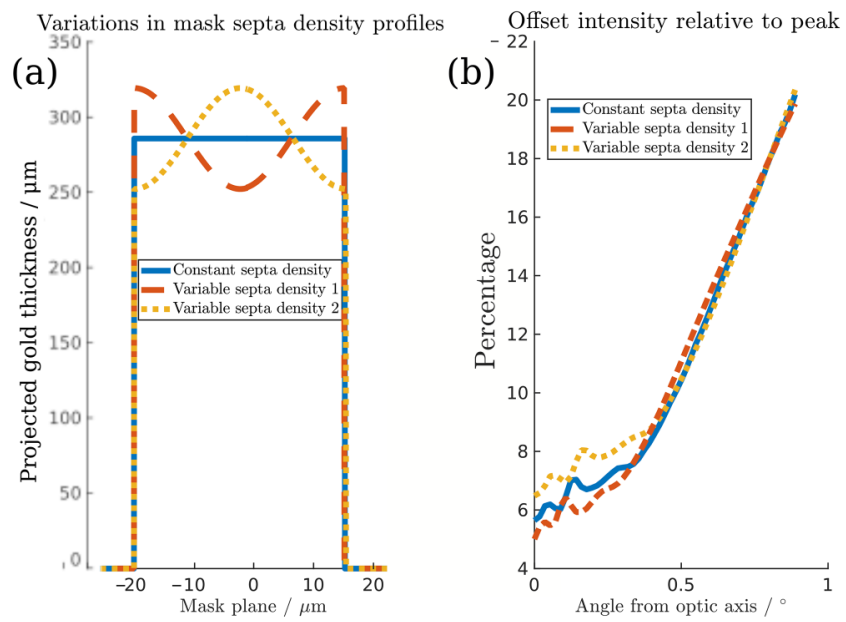

FIG. 5. The simulated effect of different septa density profiles on the IC offset intensity. (a) shows the posited gold thickness profiles and (b) the resulting offset intensities as a function of the photon angle from the optic axis. 
propagation, a scattering event may occur. The function that determines scattering does so by comparing a randomly generated number with the probability of scattering through a given volume of air, both coherently,

$$
P(\text { coherent })=1-\exp \left(\mathrm{s}_{\mathrm{i}} \rho \sigma_{\text {coh }}(E)\right)
$$

and incoherently,

$$
P(\text { incoherent })=1-\exp \left(\mathrm{s}_{\mathrm{i}} \rho \sigma_{\text {inc }}(E)\right),
$$

where $s_{\mathrm{i}}$ is the ith propagation distance, $\rho$ is the density of air, and $\sigma_{c o h}$ and $\sigma_{i n c}$ are the coherent and incoherent scattering cross sections, respectively, obtained from the NIST database. The probability of scattering, either coherently or incoherently, through $0.4 \mathrm{~m}$ of air (the separation between M1 and M2) is between $0.1 \%$ and $0.8 \%$ for the energy ranges being considered. In the event of scattering, cumulative density functions, described by Beni et al., ${ }^{27}$ are used to determine the new photon trajectory. The introduction of these adapted air-block components had negligible effects on the IC across the whole FoV, and, while lower energy settings may measure some more moderate effects, it appears that air scattering may be safely neglected at the relatively high $\mathrm{x}$-ray energies considered in this paper.
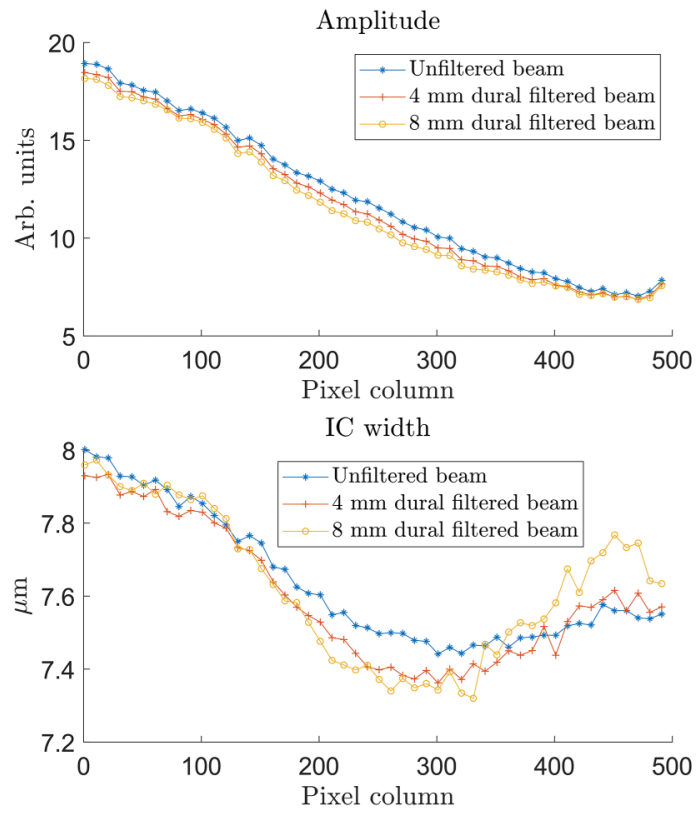

\section{MODELING OF THE ENTIRE FIELD OF VIEW AND INCLUSION OF A WIRE SAMPLE}

IC fitting considers an area-normalized Gaussian plus an offset parameter,

$$
\mathrm{IC}=a^{*} \exp \left(-\frac{(x-b)^{2}}{2 c^{2}}\right) / \sqrt{ }\left(2 \pi c^{2}\right)+d,
$$

where $x$ is the lateral position of M1, $a$ describes the IC's amplitude, $b$ its center position, $c$ its width, and $d$ the offset from zero. Figures 6(a)-6(d) show these parameters from the experimental data, with (e) showing the ratio of the offset-to-peak intensity. The variation in these retrieved parameters allows for a more complete understanding of the problems hinted at in Fig. 1.

Angular filtration is the dominant effect when considering the amplitude in Fig. 6(a). Panel (b) chiefly illustrates that the system is well aligned, with two curves corresponding to approximately the same alignment and the third being displaced by slightly less than a micron in $x$. For any given filtration, the IC centers are within a $300 \mathrm{~nm}$ range; hence, none of the effects shown are due to Moiré fringes or other alignment errors. Parts (c) and (d) show how the changing energy and effective aperture size, source width, and detector response significantly affect the IC width and offset parameters.

Fully modeling the FoV such that each of these parameters is reproduced perfectly would require more knowledge than is feasible to acquire, such as the exact heel effect distribution, the fully threedimensional density profile of the mask septa, and a perfect description of the detector response. However, by combining all of

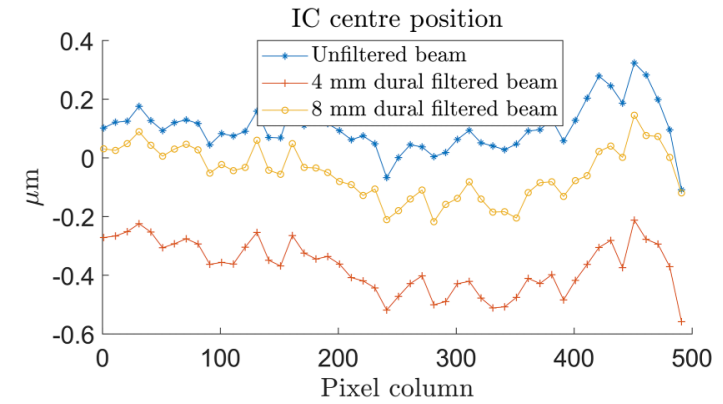

Offset relative to peak intensity

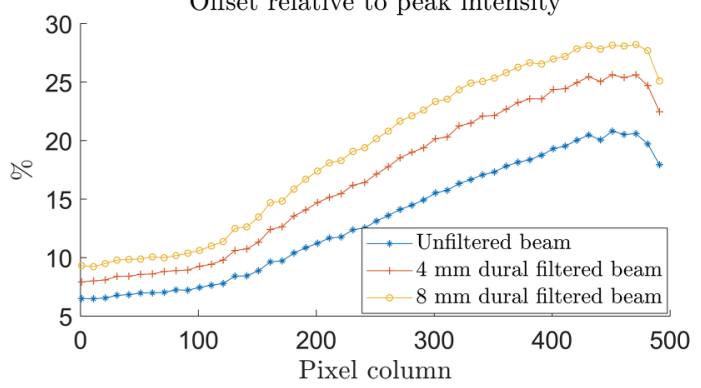

FIG. 6. Experimentally measured IC fit parameters for ICs between those highlighted in Figs. 1(e) and 1(f), averaged over each group of ten horizontal pixels for the unfiltered, $4 \mathrm{~mm}$ dural filtered and $8 \mathrm{~mm}$ dural filtered cases. (a) shows the IC amplitude, (b) the center, (c) the width parameter, and (d) the ratio of offset to peak intensity. 
the described model refinements, we achieved a reasonable reproduction of the experimentally observed behavior, as shown in Fig. 7 for the unfiltered and $8 \mathrm{~mm}$ dural filtered cases. These plots utilize the same format as in Fig. 6 and show directly how the new models provide a better agreement with that data.

Deviations between the experiment and the simulation likely occur due to uncertainty in the optic axis' exact position and the non-uniform septa thickness across the masks, which would have the greatest effect on the IC amplitude. Some notable differences can also be observed between the two models: McXtrace implements refraction of photons within the mask components, which changes the attenuation of photons and chiefly affects the offset at the higher angles of incidence; it also implements changing flux due to the heel effect, not merely the change in the average energy.

Possibly most significant, the spectral changes due to the interplay of angular filtration, detector response, and heel effect will affect the imaging results as well as the IC parameters. To experimentally reproduce this, we imaged a sapphire wire of $125 \mu \mathrm{m}$ radius at the $\pm 50 \%$ positions on the IC and retrieved the (a)

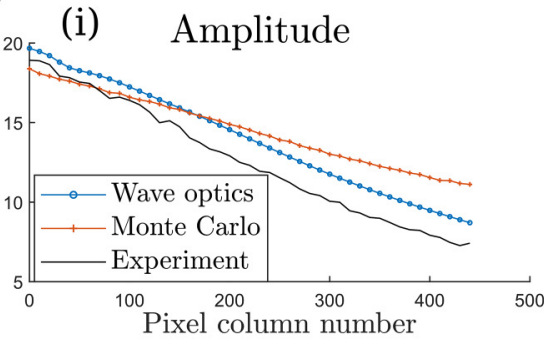

\section{Unfiltered $60 \mathrm{kVp}$ beam}
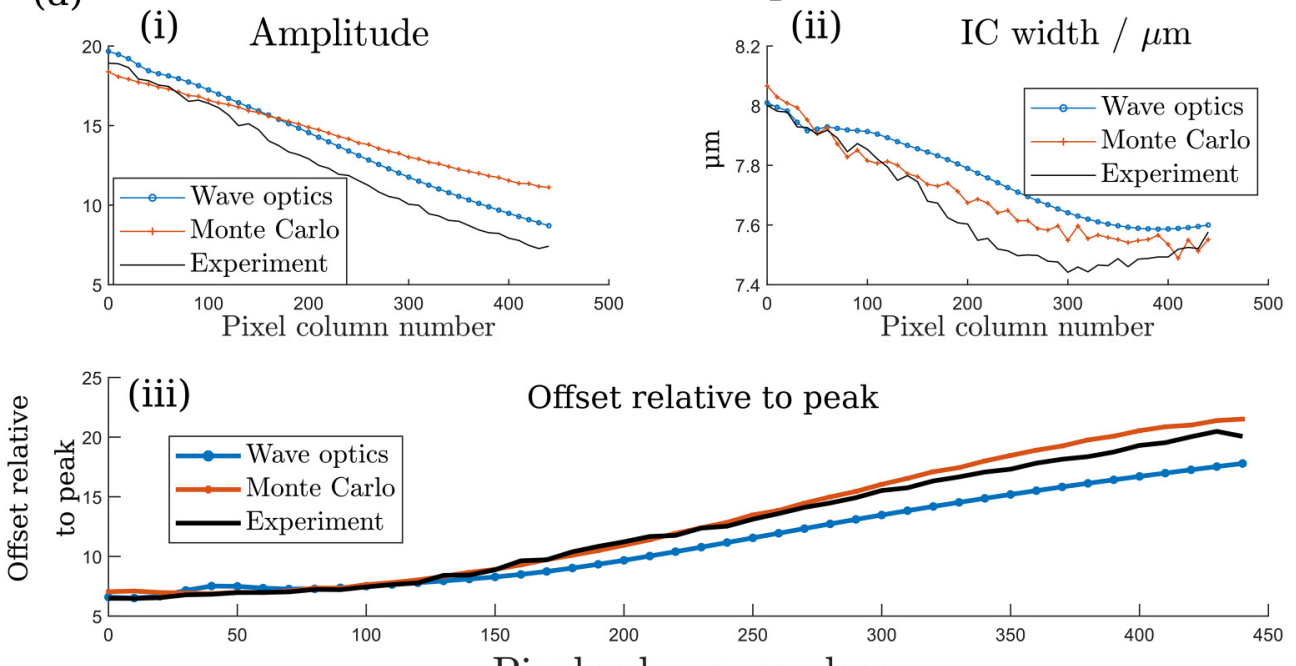

Pixel column number

(b)

$8 \mathrm{~mm}$ dural filtered $60 \mathrm{kVp}$ beam
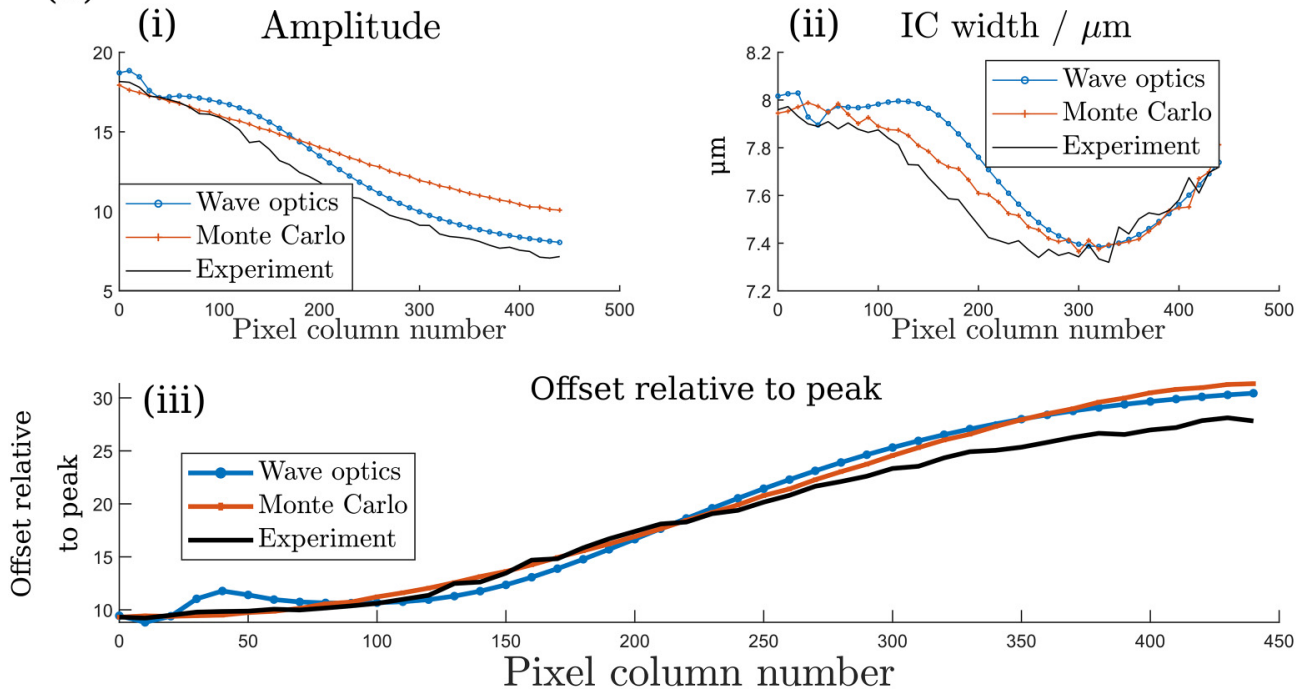

FIG. 7. Simulated IC parameters for the unfiltered (a) and $8 \mathrm{~mm}$ dural filtered (b) settings. The experimental parameters for these cases are superimposed on each plot. 


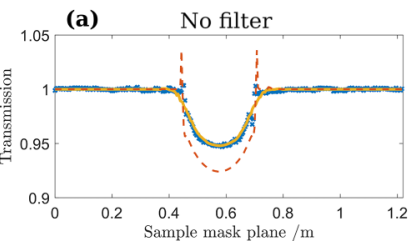

(e)

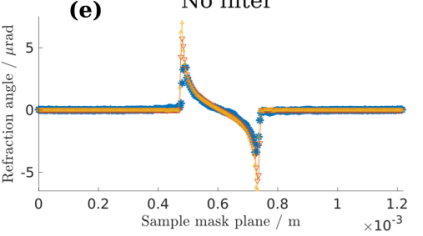

$\times$ Experimental data

$-5 \mu \mathrm{m}$ tungsten filtered refined mode

- - $5 \mu \mathrm{m}$ tungsten filtered basic model

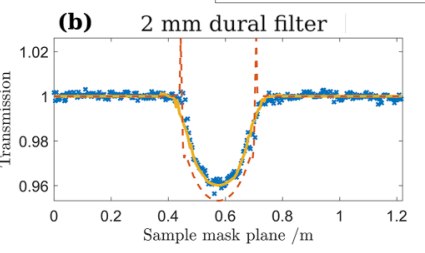

(f)
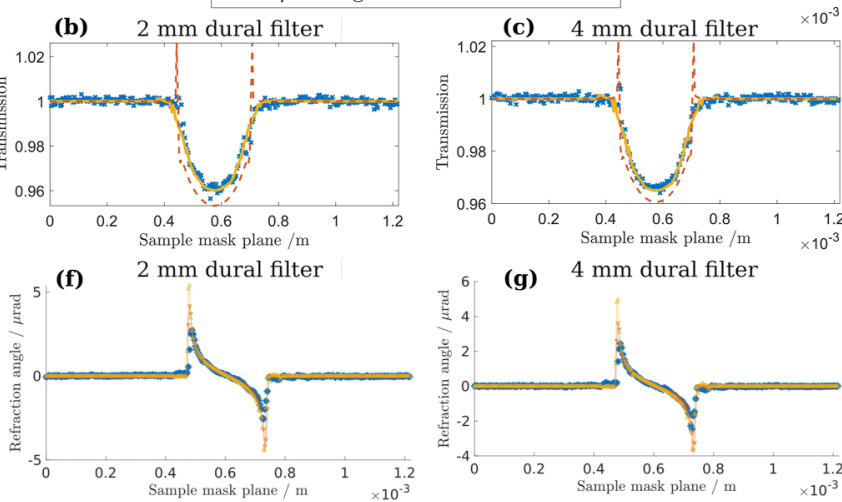

(g)

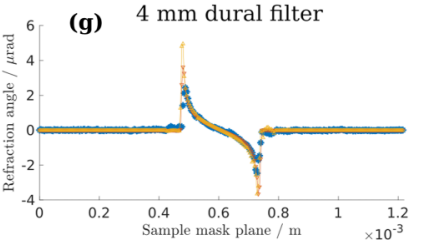

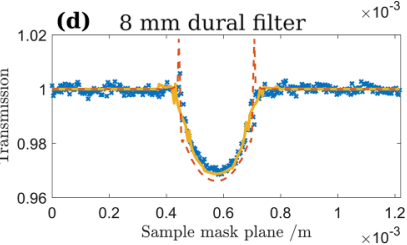

(h) $8 \mathrm{~mm}$ dural filter

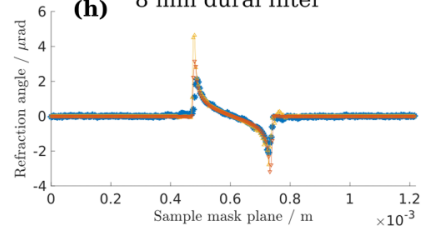

FIG. 8. Sapphire wire profiles acquired with a $60 \mathrm{kVp} \mathrm{W}$ spectrum filtered with various thicknesses of dural. Top row, (a)-(d) are retrieved attenuation profiles, Bottom row, (e)-(h), are retrieved refraction angles.

transmission and refraction signals using the R-function method developed by Diemoz et al. ${ }^{9}$ The setup was simulated using the basic and refined models, with $5 \mu \mathrm{m}$ of tungsten filtration in both cases due to the positioning of the wire, and the predictions of both WO models are compared against the experimental results in Fig. 8. MC simulations show very similar results but are not shown in order to avoid duplication and clutter.

The top row in Fig. 8 shows that attenuation is overestimated by the basic models and correctly predicted by the refined model, though the former's error decreases as the overall beam filtration increases making the spectrum harder. Notably, the refined model predicts attenuation correctly at all filtration levels. Refraction profiles are less affected by the implemented model modifications, with both the basic and refined models tending to overestimate the magnitude of the refraction peaks; this is likely due to imperfect sampling of the wire in the experimental case. Accuracy improvements in modeled attenuation will be particularly useful for techniques that rely on consistent ratios between the real and imaginary decrements from unity for materials' refractive indices. ${ }^{28}$

\section{CONCLUSION}

We have presented a series of refinements required by highenergy implementations of both Monte Carlo and wave optics models of edge illumination $\mathrm{x}$-ray phase contrast imaging. These were implemented within existing theoretical frameworks and account for the effects of angular filtration, non-linear detector response, and variation in the source size and the flux for different viewing angles. We have also shown that additional, more specific, parameters can be incorporated if needed, such as non-uniform mask septa density profiles. These additional layers of complexity introduced in the models were not found to excessively increase computational times: equivalent IC simulations saw a $22 \%$ increase in computation time for the WO case and $12.5 \%$ increase for the MC case. The improved accuracy in reproducing experimental effects, and increased understanding of previously neglected effects at high energy, will be useful when designing the next generation of EI optics. A possible avenue for future research could use these models to investigate the effects of generalized mask imperfections on system performance, and some of the effects considered and coded here may also be useful in GI studies. In the latter case, angular filtration is likely to be of concern both in terms of flux reduction and for potentially affecting the interference patterns being created.

\section{ACKNOWLEDGMENTS}

This work was funded by the Engineering and Physical Sciences Research Council (EPSRC) (Grant Nos. EP/M507581/1, EP/M028100/1, and EP/T005408/1). M.E. was supported by the Royal Academy of Engineering under the RAEng Research Fellowship scheme. A.O. was supported by the Royal Academy of Engineering under their Chairs in Emerging Technologies scheme. The support of the Karlsruhe Nano Micro Facility, a Helmholtz Research Infrastructure, through the proposal 2018-019021476 is acknowledged. The authors would like to thank Thomas Millard and Fabio Vittoria for fruitful discussions.

\section{DATA AVAILABILITY}

The data that support the findings of this study are available from the corresponding author upon reasonable request.

\section{REFERENCES}

${ }^{1}$ T. Thüring, M. Abis, Z. Wang, C. David, and M. Stampanoni, "X-ray phasecontrast imaging at $100 \mathrm{keV}$ on a conventional source," Sci. Rep. 4, 5198 (2015).

${ }^{2}$ F. Horn, M. Leghissa, S. Kaeppler, G. Pelzer, J. Rieger, M. Seifert, J. Wandner, T. Weber, T. Michel, C. Riess, and G. Anton, "Implementation of a Talbot-Lau interferometer in a clinical-like c-arm setup: A feasibility study," Sci. Rep. 8, 2325 (2018).

${ }^{3}$ L. B. Gromann, F. De Marco, K. Willer, P. B. Noël, K. Scherer, B. Renger, B. Gleich, K. Achterhold, A. A. Fingerle, D. Muenzel, S. Auweter, K. Hellbach, 
M. Reiser, A. Baehr, M. Dmochewitz, T. J. Schroeter, F. J. Koch, P. Meyer, D. Kunka, J. Mohr, A. Yaroshenko, H.-I. Maack, T. Pralow, H. van der Heijden, R. Proksa, T. Koehler, N. Wieberneit, K. Rindt, E. J. Rummeny, F. Pfeiffer, and J. Herzen, "In-vivo X-ray dark-field chest radiography of a pig," Sci. Rep. 7(1), 4807 (2017).

${ }^{4}$ D. Paganin, Coherent X-Ray Optics (Oxford University Press, 2006).

${ }^{5}$ T. P. Millard, M. Endrizzi, N. Everdell, L. Rigon, F. Arfelli, R. H. Menk, E. Stride, and A. Olivo, "Evaluation of microbubble contrast agents for dynamic imaging with X-ray phase contrast," Sci. Rep. 5(1), 12509 (2015).

${ }^{6} \mathrm{P}$. Meyer and J. Schulz, "Deep x-ray lithography," in Micro-manufacturing Engineering and Technology: Micro and Nano Technologies, edited by Y. Qin, 2nd ed. (William Andrew Publishing, Boston, 2015), Chap. 16, pp. 365-391.

${ }^{7}$ P. Meyer, T. Beckenbach, F. An, T. Schröter, J. Schulz, and J. Mohr, X-ray Gratings for Grating-Based X-ray DPCI Fabricated Using the Deep X-ray Lithography Process: State of the Art (2017).

${ }^{8}$ M. Endrizzi, P. C. Diemoz, T. P. Millard, J. Louise Jones, R. D. Speller, I. K. Robinson, and A. Olivo, "Hard x-ray dark-field imaging with incoherent sample illumination," Appl. Phys. Lett. 104(2), 3-6 (2014).

${ }^{9}$ P. C. Diemoz, F. A. Vittoria, C. K. Hagen, M. Endrizzi, P. Coan, A. Bravin, U. H. Wagner, C. Rau, I. K. Robinson, and A. Olivo, "A single-image retrieval method for edge illumination $\mathrm{x}$-ray phase-contrast imaging: Application and noise analysis," Phys. Med. 32(12), 1759-1764 (2016).

${ }^{10}$ I. Buchanan, A. Mittone, A. Bravin, P. Diemoz, M. Endrizzi, and A. Olivo, "Simplified retrieval method for edge illumination $\mathrm{x}$-ray phase contrast imaging allowing multi-modal imaging with fewer input frames," Opt. Express 28(8), 11597-11608 (2020).

${ }^{11}$ F. A. Vittoria, P. C. Diemoz, M. Endrizzi, L. Rigon, F. C. Lopez, D. Dreossi, P. R. T. Munro, and A. Olivo, "Strategies for efficient and fast wave optics simulation of coded-aperture and other x-ray phase-contrast imaging methods," Appl. Opt. 52, 6940-6947 (2013).

${ }^{12}$ T. P. Millard, M. Endrizzi, P. C. Diemoz, C. K. Hagen, and A. Olivo, "Monte Carlo model of a polychromatic laboratory based edge illumination $\mathrm{x}$-ray phase contrast system," Rev. Sci. Instrum. 85(5), 053702 (2014).

${ }^{13}$ A. M. Hernandez and J. M. Boone, "Tungsten anode spectral model using interpolating cubic splines: Unfiltered $\mathrm{x}$-ray spectra from $20 \mathrm{kV}$ to $640 \mathrm{kV}$," Med. Phys. 41(4), 042101 (2014).

${ }^{14}$ T. Schoonjans, A. Brunetti, B. Golosio, M. Sanchez Del Rio, V. A. Sole, C. Ferrero, and L. Vincze, "The xraylib library for $\mathrm{x}$-ray-matter interactions. Recent developments," Spectrochim. Acta, Part B 66(11-12), 776-784 (2011).

${ }^{15}$ P. A. Kohl, "Electrodeposition of gold," in Modern Electroplating, edited by M. Schlesinger and M. Paunovic (John Wiley \& Sons, Inc., Hoboken, NJ, 2011), pp. 115-130.
${ }^{16} \mathrm{G}$. Holmbom and B. E. Jacobson, "Nucleation and initial growth of pulseplated gold on crystalline and amorphous substrates," J. Electrochem. Soc. 135(11), 2720-2725 (1988).

${ }^{17} \mathrm{H}$. Angerer and N. Ibl, "On the electrodeposition of hard gold," J. Appl. Electrochem. 9(2), 219-232 (1979).

${ }^{18}$ R. Bellazzini, G. Spandre, A. Brez, M. Minuti, M. Pinchera, and P. Mozzo, "Chromatic x-ray imaging with a fine pitch CdTe sensor coupled to a large area photon counting pixel ASIC," J. Instrum. 8(02), C02028 (2013).

${ }^{19}$ E. Knudsen, A. Prodi, J. Baltser, M. Thomsen, P. Willendrup, M. Sánchez del Ro, C. Ferrero, E. Farhi, K. Haldrup, A. Vickery, R. Feidenhans'l, K. Mortensen, M. Nielsen, H. F. Poulsen, S. Schmidt, and K. Lefmann, "McXtrace: A Monte Carlo software package for simulating x-ray optics, beamlines and experiments," J. Appl. Crystallogr. 46, 679-696 (2013)

${ }^{20}$ A. Erko, V. Arkadiev, A. Bjeoumikhov, A. Antonov, B. Beckhoff, I. Grigorieva, H. B. Kanngießer, and B. Vidal, X-Ray Optics (Springer, Berlin, 2006), pp. 85-198.

${ }^{21}$ P. R. T. Munro, "Rigorous multi-slice wave optical simulation of $\mathrm{x}$-ray propagation in inhomogeneous space," J. Opt. Soc. Am. A 36(7), 1197-1208 (2019)

${ }^{22}$ A. Vincenzi, P. L. de Ruvo, P. Delogu, R. Bellazzini, A. Brez, M. Minuti, M. Pinchera, and G. Spandre, "Energy characterization of pixirad-1 photon counting detector system," J. Instrum. 10(04), C04010 (2015).

${ }^{23}$ K. Iniewski, H. Chen, G. Bindley, I. Kuvvetli, and C. Budtz-Jørgensen, Modeling Charge-Sharing Effects in Pixellated CZT Detectors (IEEE, 2007) Vol. 6, pp. 4608-4611.

${ }^{24}$ S. Awadalla, Solid-State Radiation Detectors, 1st ed. (CRC Press, 2017).

${ }^{25}$ P. C. Diemoz, C. K. Hagen, M. Endrizzi, and A. Olivo, "Sensitivity of laboratory based implementations of edge illumination x-ray phase-contrast imaging," Appl. Phys. Lett. 103(24), 244104 (2013).

${ }^{26}$ M. Busi, U. L. Olsen, E. B. Knudsen, J. R. Frisvad, J. Kehres, E. S. Dreier, M. Khalil, and K. Haldrup, "Simulation tools for scattering corrections in spectrally resolved x-ray computed tomography using McXtrace," Opt. Eng. 57(3), 037105 (2018).

${ }^{27}$ M. S. Beni, D. Krstic, D. Nikezic, and K. N. Yu, "Monte Carlo studies on photon interactions in radiobiological experiments," PLOS One 13(3), e0193575 (2018).

${ }^{28}$ P. C. Diemoz, F. A. Vittoria, C. K. Hagen, M. Endrizzi, P. Coan, E. Brun, U. H. Wagner, C. Rau, I. K. Robinson, A. Bravin, and A. Olivo, "Single-image phase retrieval using an edge illumination $\mathrm{x}$-ray phase-contrast imaging setup," J. Synchrotron Radiat. 22(4), 1072-1077 (2015). 